\title{
The Reduction of Ventrolateral Prefrontal Cortex Gray Matter Volume Correlates with Loss of Economic Rationality in
} Aging

\author{
난-Kuan Chung, ${ }^{1}$ Agnieszka Tymula, ${ }^{3,4}$ and Paul Glimcher ${ }^{2,4}$ \\ ${ }^{1}$ Department of Psychology, ${ }^{2}$ Center for Neural Science, New York University, New York, New York 10003, ${ }^{3}$ School of Economics, University of Sydney, \\ Sydney 2006, New South Wales, Australia, and ${ }^{4}$ Institute for the Study of Decision Making, Brooklyn, New York 11201
}

The population of people above 65 years old continues to grow, and there is mounting evidence that as humans age they are more likely to make errors. However, the specific effect of neuroanatomical aging on the efficiency of economic decision-making is poorly understood. We used whole-brain voxel-based morphometry analysis to determine where reduction of gray matter volume in healthy female and male adults over the age of 65 years correlates with a classic measure of economic irrationality: violations of the Generalized Axiom of Revealed Preference. All participants were functionally normal with Mini-Mental State Examination scores ranging between 26 and 30. While our elders showed the previously reported decline in rationality compared with younger subjects, chronological age per se did not correlate with rationality measures within our population of elders. Instead, reduction of gray matter density in ventrolateral prefrontal cortex correlates tightly with irrational behavior. Interestingly, using a large fMRI sample and meta-analytic tool with Neurosynth, we found that this brain area shows strong coactivation patterns with nearly all of the value-associated regions identified in previous studies. These findings point toward a neuroanatomic locus for economic rationality in the aging brain and highlight the importance of understanding both anatomy and function in the study of aging, cognition, and decision-making.

Key words: aging; brain structure; economic rationality; generalized axiom of revealed preference; ventrolateral prefrontal cortex; voxel-based morphometry

\section{Significance Statement}

Age is a crucial factor in decision-making, with older individuals making more errors in choices. Using whole-brain voxel-based morphometry analysis, we found that reduction of gray matter density in ventrolateral prefrontal cortex correlates with economic irrationality: reduced gray matter volume in this area correlates with the frequency and severity of violations of the Generalized Axiom of Revealed Preference. Furthermore, this brain area strongly coactivates with other reward-associated regions identified with Neurosynth. These findings point toward a role for neuroscientific discoveries in shaping long-standing economic views of decision-making.

\section{Introduction}

Previous studies have shown that even high-functioning older adults achieve lower rates of return when choosing over financial

Received April 28, 2017; revised Sept. 27, 2017; accepted Sept. 28, 2017.

Author contributions: H.-K.C., A.T., and P.G. designed research;H.-K.C. performed research;H.-K.C., A.T., and P.G. analyzed data; H.-K.C., A.T., and P.G. wrote the paper.

This study was funded by the National Institutes of Health (Grant NIH-NINDS-NS-054775 to P.G.). We thank Michael Grubb for assistance with participants recruitment and MRI scanning, Gregory Samanez-Larkin for advice on Digit Span and Shipley Vocabulary tests, Dan Burghart for assistance in programming GARP analysis, Joshua Riepe for assistance with data collection and North American Adult Reading Test analysis, Keith Sanzenbach for assistance with MRI scanning, and Benjamin Lu for helpful feedback on this manuscript.

The authors declare no competing financial interests.

Correspondence should be addressed to Paul Glimcher, Center for Neural Science, New York University, 4 Washington Place, Room 809, New York, NY 10003, E-mail: paulg@nyu.edu.

DOI:10.1523/JNEUROSCI.1171-17.2017

Copyright $\odot 2017$ the authors $\quad 0270-6474 / 17 / 3712068-10 \$ 15.00 / 0$ lotteries than do their younger counterparts (Tymula et al., 2013). This appears to be true even though one's knowledge base increases with age, giving older adults an advantage in some situations (Li et al., 2013). Older adults demonstrate less effective performance in multiple financial datasets (Agarwal et al., 2009) and higher frequencies of misvoting in the political arena (Shue and Luttmer, 2009). Since older people remain in the workforce longer and thus continue to make decisions that affect them and societies, it is important to understand the neural underpinnings of rationality decline in aging.

Economic theory has formalized a precise definition of "rationality" that we seek to leverage here. Technically, rational decision makers are those whose choices can be shown to be consistent with maximization of utility, a subjective quantity meant to represent an individual's "satisfaction level." Obeying the General Axiom 


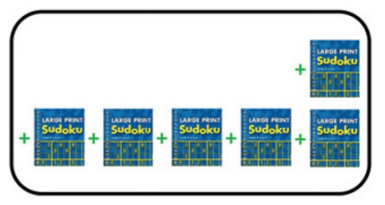

7. Crossword:0

Sudoku:6

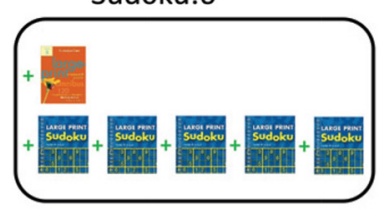

4. Crossword:1

Sudoku:5

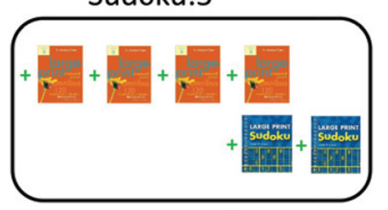

1. Crossword:4

Sudoku:2

\section{I will GAIN}

? Crossword book and ? Sudoku book !

\section{Press numerical keyboard to select the option}

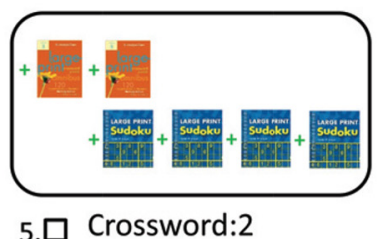

5. Crossword:2

Sudoku:4

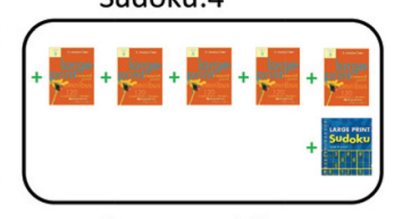

2. Crossword:5

Sudoku:1

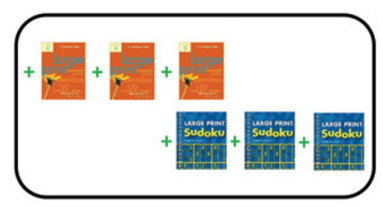

6. Crossword:3

Sudoku:3

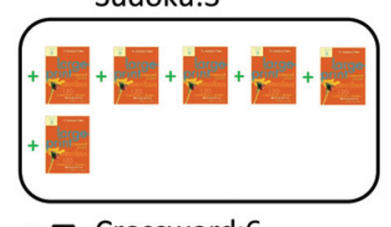

3. Crossword:6

Sudoku:0 relating neuroanatomy to economic rationality in otherwise healthy elderly subjects.

In this article, we examined whether brain structure correlates with experimentally derived estimates of economic irrationality (GARP violations) in a population of elders. We found that elders show a decline in choice rationality when compared with other groups reported in the literature. However, we did not find a statistically significant correlation between age and rationality within our narrow age band (65-92 years old), but instead we found a correlation between GM density in ventrolateral prefrontal cortex (mainly a47r in the Human Connectome Project; Glasser et al., 2016) and the frequency and the severity of economic irrationality. A meta-analysis with Neurosynth (Yarkoni et al., 2011) revealed that nearly all of the regions associated with valuation and decision-making show strong coactivation patterns with this area. of Revealed Preference (GARP; Afriat, 1967) is the necessary and sufficient condition for being a utility maximizer (Samuelson, 1938; Richter, 1966; Varian, 1982). GARP says that if option X was chosen when option Y was affordable, and option Y was chosen when option $\mathrm{Z}$ was affordable, rational people should NOT prefer Z over X. In other words, choices should be internally consistent and transitive. If a person violates GARP, this means that his/her choices are not consistent with utility maximization and no reasonable utility function exists that could explain his/ her decisions. Such rationality violations could be caused by a range of factors - general propensity to make mistakes, preference instability, or distractions - all of which would result in observed preference intransitivity, hence GARP violations. Previous studies have assessed the degree of economic rationality observed in children (Harbaugh et al., 2001), during altruistic decisions (Andreoni and Miller, 2002), in drunk people (Burghart et al., 2013), in women throughout menstrual cycle (Lazzaro et al., 2016), and in patients with a frontal lobe lesion (Camille et al., 2011). Our first goal was to perform similar measurements for the first time in a group of elders.

At the same time that elders show increases in decision error rates, they also experience profound changes in their brains. $\mathrm{Nu}-$ merous studies have documented age-related alterations in the brain and cognitive functions (Hedden and Gabrieli, 2004; Bishop et al., 2010; Grady, 2012; Gutchess, 2014; Samanez-Larkin and Knutson, 2015). Grubb et al. (2016) found that the gray matter (GM) density correlates with risk preference change in aging, and chronological age has no additional predictive power once one has accounted for GM density differences. This raises the possibility that the age-related decline of choice rationality and loss of GM volume are related. In line with this logic, earlier work has shown that children, whose brains are still not fully developed (Giedd, 2004; Gogtay et al., 2004), violate GARP more frequently (Harbaugh et al., 2001). In addition, patients with frontal lobe lesions have been shown to have problems with utility maximization as defined by GARP (Camille et al., 2011). To the best of our knowledge, there are no empirical findings directly

\section{Materials and Methods}

Participants. Thirty-nine healthy individuals (age, 65-92 years; average age, 72.44 years; 14 males) participated in this study. One participant was excluded from analysis due to excessive head motion during MRI scanning. Participants were not taking medication for any psychiatric condition or developmental disorder. They had normal or corrected-tonormal vision. None of the participants tested positive for dementia in the Mini-Mental State Examination (Folstein et al., 1975); all participants scored between 26 and 30 (mean, 29.05). The average intelligence score (mean, 42.53 on the North American Adult Reading Test; Blair and Spreen, 1989) in our sample is not significantly different $\left(t_{(37)}=-0.42\right.$, $p=0.68)$ from a similar previous study with a larger sample of elders $(N=122 ;$ mean, 43.23;Uttl, 2002). Participants were recruited via advertising in local newspapers, digital newsletters, a departmental website, and flyers on bulletin boards on the New York University Washington Square campus and at nearby community centers. The data were collected at Washington Square campus of New York University. The University Committee on Activities Involving Human Subjects at New York University approved this study.

Experimental design. To quantify the degree of technical rationality in each subject, we adopted a behavioral paradigm designed by Harbaugh et al. (2001) in which people are asked to select their favorite bundle of two goods from three to seven different alternatives. Figure 1 shows an example of a screenshot from the experiment that consists of seven alternatives. In this example, there are seven options (bundles), and each option (bundle) consists of different numbers of crossword books and sudoku books. In this example, books trade off one-for-one-to have one more crossword book, the participant needs to give up one sudoku book. Participants were instructed to select the one bundle that they liked the most from those displayed on the screen by pressing the corresponding number on the numeric keyboard. For example, in Figure 1, if they preferred the bundle with two crossword books and four sudoku books, they would need to press 5 . After each selection, subjects were told to double-check their answer (which could be changed at that point) before they pressed "Enter" to continue to the next trial.

In total, there were 11 different choice sets (11 unique trials, each presented only once) in the experiment. We visualize them all in Figure $2 A$, with dots representing bundles (options) and lines representing choice sets (trials). The red line shows the example presented in Figure 1. Some of the lines are steeper than others, which captures differences in 

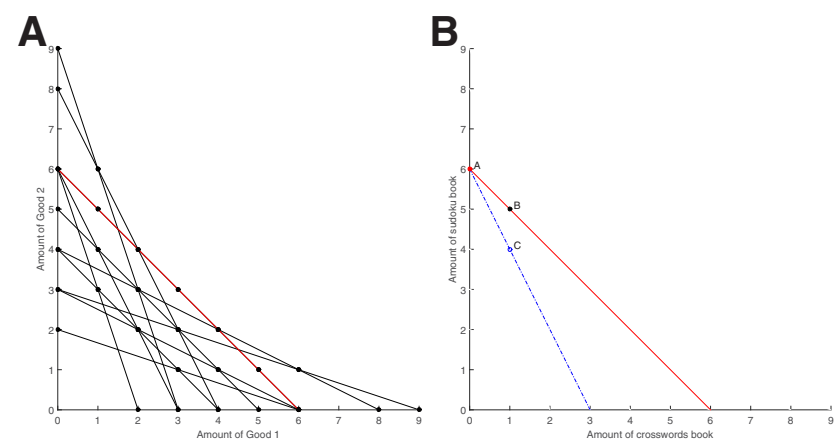

Figure 2. $\quad A$, Eleven choice sets in the experiment, with dots representing bundles (options) and lines representing choice sets (trials). The red line shows the example presented in Figure 1. $B$, One example of choices that violate GARP from a simulated participant. The selected bundle $A$ is directly revealed preferred to all other bundles on the red solid line including bundle B. By monotonicity, bundle $B$ is objectively better than the corresponding bundle on the blue dashed line (bundle C). By transitivity, bundle A is preferred to bundle C. However, the simulated participant in our example selected bundle C (blue dot), a choice that violates GARP.

the relative tradeoffs between the two classes of goods. The slope of the red line is equal to -1 ; moving down the line, every time the number of sudoku books decreases by one, the number of crossword books increases by one. If the slope is steeper, the number of sudoku books one needs to surrender to get one more crossword book increases. In our steepest choice set, one crossword book is worth three sudoku books (economists interpret these choice sets as fixed budgets, income constraints, with different relative prices for each of the bundle elements). The order of presentation of the 11 choice sets was randomized independently for each participant. At the end of the experiment, each participant was paid according to his/her choice in one randomly selected trial. Since every choice had a positive probability of being realized, the choosers had an incentive to reveal their true preferences in each of their choices. The 11 choice sets in our study are informative enough to assess rationality violations and have been validated in a number of previous studies (Harbaugh et al., 2001; Andreoni and Miller, 2002; Camille et al., 2011; Burghart et al., 2013; Lazzaro et al., 2016). This paradigm is simple to understand, does not involve any complicated calculations, and is quick to implement (only 11 trials), which helps maintain participants' attention.

Intuitively, the type of reward used in the experiment can influence an individual's rationality score. In an effort to improve our ability to correctly detect a participant's irrationality in choice, we implemented three slight modifications relative to the original study by Harbaugh et al. (2001) in which the bundles always consisted of the same goods, juice and chips, for all subjects. First, we wanted the participants to care about the choices they made, which means that they should want to possess the goods that constitute the bundles on offer. If individuals were not interested in the products that they were choosing between, we might mistakenly interpret complete randomness in choice (due to not caring) as irrationality. To overcome this problem, we selected a diverse range of small and easily portable consumer goods attractive to elders: crossword books, sudoku books, pens, hand cream, sanitizer, pocket tissues, oneway MTA (mass transit) tickets, tea bags, chocolates, instant noodle cups, granola bites, and breathe mints. We asked each individual participant to report the desirability of each of the products on an 8-point scale. We also told them in advance that the maximum quantity of any one good they could earn from the experiment was 9 units, and that they should consider this when reporting the desirability of each good. We conducted the desirability ranking twice, once when the individual scheduled the appointment and a second time on the day of the experiment, right before the task. For each of the participants, we used only two types of goods that they rated as relatively desirable on the day of the experiment. Since people usually do not want to have more than one of the same crossword or sudoku books, we prepared multiple items in these two (popular) categories consisting of different volumes of crossword and sudoku books. For these goods, we told participants that the picture on the screen was just a representative of a category and that each crossword book that they took home would be different. With these procedures, we assume that having more of a good was always better than having less of that good (strong monotonicity) within the quantity range offered in the experiment. Note that by design we need to strengthen the original requirements of weak monotonicity and local nonsatiation in GARP to strong monotonicity. This strong monotonicity rules out satiation and indifference to quantity change. We adopt this approach for simplicity, and the interested reader can turn to the studies by Glimcher (2010), Varian (1982), or Kreps (1990) for a more detailed explanation of axioms of technically rational choices.

Second, since each bundle consisted of some quantity of two different goods, and when making their choice people trade off the quantity of one good for the other good, we wanted to select these goods such that one of them is not obviously better than the other one. If one of the goods in the bundle is strongly preferred to the other one, people will use a simple strategy and always choose a bundle with more of their strongly preferred good. In our test, it will give us little power to assess a participant's actual ability to be rational. Therefore, for each participant we customized the bundles with two products that this participant not only liked but also ranked as approximately equally desirable.

Third, participants were allowed unlimited time to fully consider all options before making a decision, and they could also alter their answer until they continued to the next trial (at which point they could not go back). The irrational decisions that we observed should not be due to time pressure.

Behavioral analysis: rationality measurement. Recall that if an individual chooses bundle A when bundles A and B are offered and he/she chooses $B$ when $B$ and $C$ are offered, then when offered a choice between $\mathrm{A}$ and $\mathrm{C}$, to obey GARP, he/she must choose A. To understand how a participant can violate GARP in our task, consider the choices from one simulated participant presented in Figure $2 B$. This participant violated GARP because he/she selected A from the red solid choice set and C from the blue dashed choice set. Since the bundle A was selected from the red solid choice set, we can conclude that A was directly revealed preferred to all other bundles on the red solid line. Now notice that by monotonicity (more is better, as built into our design) there is no bundle on the blue line that could be considered objectively better than a corresponding bundle (i.e., with the same quantity of crossword books) on the red solid line. For example, bundle B is objectively better than bundle $C$ because $B$ has more sudoku books and the same quantity of crossword books as C. Therefore, since bundle A was preferred to B, it should also be preferred to $C$. However, the simulated participant in our example selected bundle C (blue dot), a choice that violates GARP. It should be noted that this explanation of GARP is something of a simplification. We adopt this exposition for expediency. The interested reader can turn to the studies by Glimcher (2010) and Varian (1982) for a more detailed explanation of GARP.

Our procedure to identify choices that violate GARP goes as follows: we use an $11 \times 2$ matrix of $x=\left(x^{i}, \ldots, x^{k}\right)$ to represent the quantity of each good in the 11 bundles selected by a subject. For instance, we would describe the choices of the above simulated participant as $x^{1}=[0 ; 6]$ and $x^{2}=[1 ; 4]$. We also construct a $2 \times 11$ matrix $p=\left(p^{i}, \ldots, p^{k}\right)$ of the relative prices of the associated goods, normalizing the price of the good one to one. For example, in our red solid budget set, giving up one crossword book gives one extra sudoku book, thus the relative prices are $p^{1}=\left[\begin{array}{ll}1 & 1\end{array}\right]$. In the blue dashed budget set, giving up one crossword book gives two extra sudoku books, thus the relative prices are $p^{2}=\left[\begin{array}{ll}1 & 0.5\end{array}\right]$. Note that relative prices are equivalent to the slopes of the budget sets in Figure $2 A$. We can then formulate the definition of GARP following the study by Varian (1982) as follows: if there is a preference relation for $x^{i}$ and $x^{k}$ written as $x^{i} \boldsymbol{R} x^{k}\left(\boldsymbol{R}\right.$ denotes that $x^{i}$ is revealed preferred to $\left.x^{k}\right)$ from some sequence of observations $x^{i}, \ldots, x^{k}$ that $p^{i} x^{i} \geq p^{i} x \geq p^{i} x^{k}$, it does not imply $p^{k} x^{k}>p^{k} x^{i}$ ( $x^{k}$ is not strictly directly revealed preferred to $x^{i}$ ).

Mathematically, we can use Warshall's algorithm (Warshall and Stephen, 1962; Varian, 1996) to obtain the whole transitive closure of the observed choices. First, we construct an $11 \times 11$ matrix M, by which $i-j$ entry is given by: 


$$
M_{i j}= \begin{cases}1 & \text { if } p^{i} x^{i} \geq p^{i} x^{j} \\ 0 & \text { otherwise }\end{cases}
$$

$\mathrm{M}$ describes the direct relation by observing selected bundles, and then we can operate Warshall's algorithm on M to create a transitive closure matrix MT as follows:

$$
M T_{i j}=\left\{\begin{array}{ll}
1 & \text { if } x^{i} R x^{j} \\
0 & \text { otherwise }
\end{array} .\right.
$$

Then, by checking whether $p^{j} x^{j}>p^{j} x^{i}$ when $M T(i, j)=1$, we can quantify GARP violations in observed choices. For instance, $M T(2,1)=1$ indicates that bundle $\mathrm{C}$ is revealed to be preferred to bundle $\mathrm{A}\left(p^{2} x^{2} \geq p^{2} x^{1}\right)$ in the blue dashed budget set. However, by observing that bundle $\mathrm{A}$ is selected from the red solid budget set, we know that bundle A is strictly directly revealed preferred to bundle $\mathrm{C}$ while bundle $\mathrm{C}$ is still affordable $\left(p^{1} x^{1}>p^{1} x^{2}\right)$. A pair of GARP violations appears, and two bundles are identified that violate GARP in this case.

In this report, we use two measures to quantify the level of irrationality of our choosers, as follows: (1) the frequency of irrationality that we calculate as the number of selected bundles that violated GARP; and (2) the severity of irrationality captured by the Houtman-Maks (HM) index (Houtman and Maks, 1985). The HM index captures the notion that if the violation results in a small loss, it should not be assessed as severe. The HM index achieves this by counting the number of (erroneous) choices that would need to be removed from the observed set of choices to make the chooser look perfectly rational. By definition, the HM index is the largest subset of all observed choices that do not include any GARP violation. The maximum HM index in this study is 11 , with a higher number indicating higher GARP consistency. We additionally performed the analysis using alternative irrationality measures-total pairs of GARP violations (Camille et al., 2011) and Afriat Efficiency Index (AEI; Afriat, 1972) in supplementary materials on personal website (http://decisionsrus.com/documents/Supplementary\%20Material\%202. pdf). This yielded qualitatively very similar results. All measures were calculated using a script in Matlab R2015a (MATLAB, MathWorks).

General procedures. Participants completed the GARP task outside the scanner after their MRI anatomical scan. They read the instructions and were given an opportunity to ask questions, answered several comprehension questions, and completed several practice rounds to get familiar with the software. Participants performed the task by themselves, one participant per each experimental session. Including preparation time and scanning, most participants finished the experiment within $2 \mathrm{~h}$ and were paid a $\$ 50$ participation fee plus task earnings. The task was programmed using E-Prime 2.0 (Psychology Software Tools).

All participants filled out a simple demographic form, including age, gender, handedness, and education level. We also measured numeracy skills using the numeracy test of the US Health and Retirement Study (Ofstedal et al., 2005), intelligence using the Shipley Vocabulary test (Shipley, 1940; Zachary and Shipley, 1986), and cognitive ability using Digit Span test (Wechsler, 1997). We were unable to collect Shipley Vocabulary and Digit Span scores for two participants. In the analysis, their scores were replaced with the average scores from the rest of 36 participants in our sample. All surveys were completed after the GARP task. The instructions and comprehension questions are available in supplementary materials on personal website (http://decisionsrus.com/documents/ Supplementary\%20Material\%202.pdf).

MRI acquisition. T1-weighted high-resolution anatomical images $(1 \times$ $1 \times 1 \mathrm{~mm}^{3}$ ) were acquired with a magnetization-prepared rapid acquisition gradient echo pulse sequence (TR, $2.5 \mathrm{~s}$; TE, $3.93 \mathrm{~ms}$; T1, $900 \mathrm{~ms}$; flip angle, $8^{\circ} ; 176$ sagittal slices; $256 \times 256$ matrix in a $256 \mathrm{~mm}$ field of view) using a $3 \mathrm{~T}$ Siemens Allegra head-only scanner equipped with a custom radiofrequency coil (NM-011 transmit head coil, NOVA) at the New York University Center for Brain Imaging.

Voxel-based morphometry analysis. Voxel-based morphometry (VBM) analysis was performed using the VBM8 toolbox (http://www.neuro. uni-jena.de/vbm/) and SPM8 (http://www.fil.ion.ucl.ac.uk/spm) on Matlab R2015a. Using the VBM8 toolbox, the structural images were segmented into gray matter, white matter, and CSF based on a modified Gaussian mixture model (Ashburner and Friston, 2000). A hidden Markov random field weighting of 0.15 was used to minimize the noise level through spatial constraints of neighboring voxels (Zhang et al., 2001; Cuadra et al., 2005). A light clean-up procedure of extracting the brain from segmentations was used through a crude routine performing conditional dilations and erosions. Images were registered to a standard stereotactic atlas [Montreal Neurological Institute (MNI)] via the Diffeomorphic Anatomical Registration Through Exponentiated Lie Algebra process (Ashburner and Friston, 2000; Ashburner, 2007). To preserve the original GM volume, a modulation of volume changes due to affine transformation (global scaling) and nonlinear warping (local volume change) was applied. Finally, the normalized-modulated GM images were convolved with a Gaussian kernel (FWHM $=8 \mathrm{~mm}$ ) using SPM8.

We modeled normalized-modulated-smoothed GM voxels with a multiple regression with irrationality as the variable of interest. In addition, several nuisance covariates were included jointly in the same model. The global GM volume, gender, age, handedness, education level, numeracy skill, Shipley verbal IQ score, digit span score, and average response time were included in the design matrix and were thus regressed out. We ran this analysis using the following two measures of irrationality: (1) the frequency of irrationality; and (2) the severity of irrationality (HM index). Due to the nonuniform smoothness of VBM data (Hayasaka et al., 2004; Kurth et al., 2015), we enabled a nonstationary cluster correction by switching the default in SPM8 to "spm_get_defaults ('stats.rft.nonstat',1)." The cluster-defining threshold was set as uncorrected $p<0.001$ at peak level. Note that this threshold has much better FWE control than a cluster-defining threshold at $p<0.01$ (Eklund et al., 2016). Masking with a 0.05 absolute threshold was applied to alleviate false-positive results outside the brain that arose due to residual variance approaching zero along the GM boundary.

Brain circuits of economic rationality in Neurosynth dataset. To examine the brain circuits related to the region identified in our VBM analysis, we next performed a meta-analysis of brain coactivation in the Neurosynth (http://www.neurosynth.org/) database with prior studies. At the time of our analyses (8 June 2017), Neurosynth included 11,406 published and peer-reviewed studies. To do this, we first used the key term "reward," using the "reverse inference" test for meta-analytic term-to-activation mappings. We also reported the result of a "forward inference" test to compare with a previous study (Bartra et al., 2013). The automated metaanalytic map was created by identifying coordinates that were consistently and preferentially reported in studies in which the term reward occurred frequently. This procedure identified 671 studies (on 8 June 2017). Voxels were converted to $z$-scores and corrected for multiple comparisons with threshold set at an expected false discovery rate of 0.01 . In other words, voxels with higher $z$-scores were reported as more likely in articles mentioning reward more often. We call this the "reward map." Second, we used the location derived from the VBM analysis above and examined functional connectivity and meta-analytic coactivation with that coordinate and various other voxels in the Neurosynth database. This coactivation map with $z$-scores indicated brain regions coactivated across the resting-state fMRI time series and also brain regions coactivated across publications in the Neurosynth within the $6 \mathrm{~mm}$ hard sphere seed centered on our coordinate of interest. Finally, we compared the coactivation map we extracted with the reward map from Neurosynth to shed light on the brain circuits related to our VBM finding.

\section{Results}

\section{Behavioral result: higher proportion of irrational decision makers in an older population}

The older adults in our study presented varying levels of irrationality in choices with $42.11 \%$ of participants making two or more GARP violations. We can cautiously compare this number to previous studies with different populations. In doing such a comparison, it is important to note that by including only desirable consumer goods, we likely made it easier to satisfy GARP than if we used the same procedures as in these other studies. Nevertheless, the proportion of irrational individuals in our sample is higher than the proportion of irrational individuals (35\%) among college undergraduates in the study by Harbaugh et al. (2001) (Fig. 3). 


\section{VMF patients}

Camille et al. (2011)

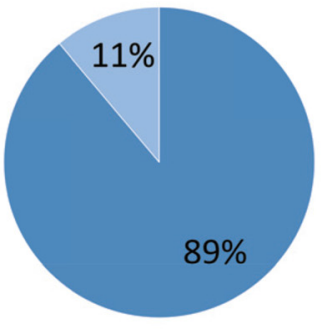

Drunk people

Burghart et al. (2013)

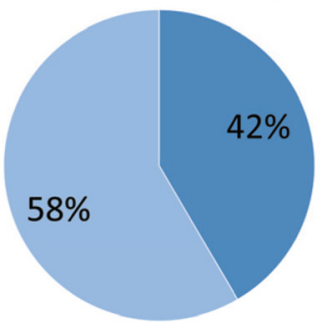

$2^{\text {nd }}$ grade children

Harbaugh et al. (2001)

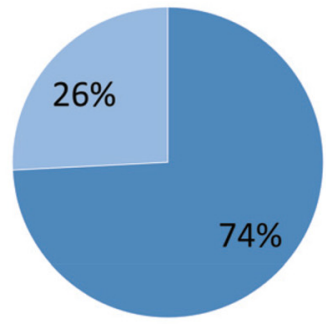

Undergraduates

Harbaugh et al. (2001)

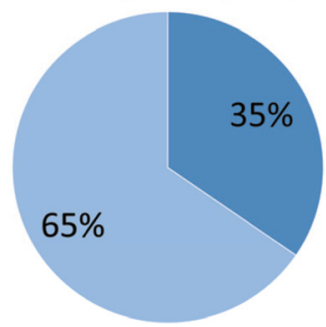

Present study

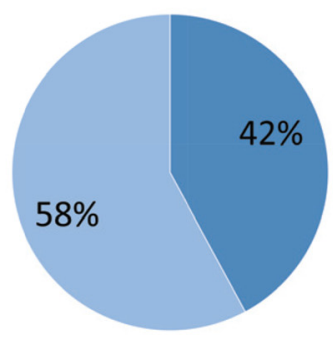

Two or more No GARP violation

Figure 3. Proportion of GARP violations in different populations in the present study and the studies by Camille et al. (2011), Harbaugh et al. (2001), and Burghart et al. (2013).

Although this difference is not significant, a number of other higher-powered studies show that elders are more irrational than their younger peers (Agarwal et al., 2009). Our elders appear to violate rationality less often than second-grade children $(74 \%$ irrational individuals in the study by Harbaugh et al., 2001) and patients with ventromedial frontal lobe lesions ( $89 \%$ of irrational individuals in Camille et al., 2011). Our participants violate rationality equally as often as younger people with blood alcohol levels at or above the $0.8 \%$ legal limit (42\% of irrational individuals in the study by Burghart et al., 2013).

We find similar results using the severity of irrationality measure (HM index). The older adults in our study presented levels of rationality (average HM index $=10.45$ ) similar to drunk people (average HM index $=10.43$ ) in the study by Burghart et al. (2013). Severity of irrationality correlated with frequency of irrationality (Pearson correlation $r_{(36)}=-0.95, p<0.001$ ).

Behavioral result: economic irrationality was not significantly correlated with age

On average, our participants made 1.37 irrational choices. The most irrational participant made six irrational choices, which means that he/she violated GARP in more than half of the choice situations. Interestingly, the frequency of irrationality (the number of selected bundles that violated GARP) is not significantly correlated with age in this small sample, despite the fact that elders appear to have a higher level of irrationality overall (Fig. $4 A$; Pearson correlation $r_{(36)}=-0.2, p=0.22$ ). What is most important for this report is that the wide distribution of the rationality index in our sample allowed us to explore the relationship between the degree of economic rationality and gray matter volume throughout the brain. The frequency of irrationality significantly correlated with Shipley verbal IQ (Pearson correlation $r_{(36)}=-0.34, p=0.03$ ) but not with the other cognitive questionnaires measured in this study. Using the HM index, we obtained a similar result that there is no significant correlation between the severity of irrationality and chronological age (Fig. $4 B$; Pearson correlation $\left.r_{(36)}=0.25, p=0.13\right)$.
A

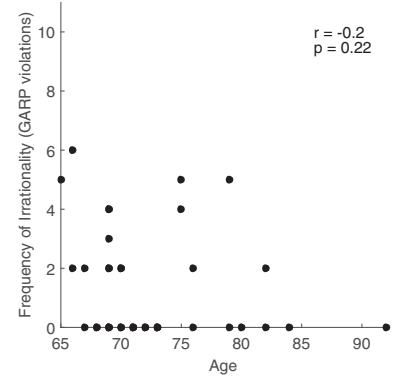

B

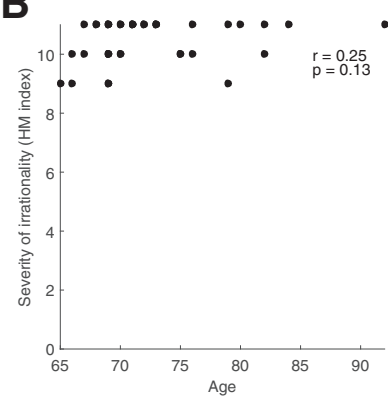

Figure 4. For each individual, age is plotted on the $x$-axis against the frequency of irrationality (the number of selected bundles that violated GARP on the $y$-axis; $\boldsymbol{A}$ ) and on the $y$-axis against the severity of irrationality (HM index; $\boldsymbol{B})$. Both were not significantly correlated with age.

VBM result: less GM in ventrolateral prefrontal cortex correlates with higher rates of economic irrationality

We used whole-brain VBM analysis to determine whether economic rationality is correlated with gray matter density. We found that the gray matter volume of a cluster in the left ventrolateral prefrontal cortex (vlPFC; lateral Brodmann's area 10; rostral Brodmann's area 46; lateral Brodmann's area 47) was negatively correlated with the frequency of economic irrationality (Fig. 5A; peak at MNI coordinates $x=-35, y=53, z=-2$; cluster number, 771 ; cluster level with FWE, $p=0.01$; cluster level with FDR, $p=0.03$ ). Participants with less GM in this region showed more GARP violations in their selected bundles (Fig. 6A). Figure $6 B$ shows the scatter plot with gray matter volume at the same locations with chronological age. This region mainly maps to area $\mathrm{a} 47 \mathrm{r}$ and extends to the rostral part of $\mathrm{p} 47 \mathrm{r}$ and $\mathrm{a} 9-\mathrm{v} 46$ and 47I, as identified by the Human Connectome Project (Glasser et al., 2016). No other region was significantly correlated with economic irrationality using the same analytic method. The cluster in left vlPFC (peak at MNI coordinates, $x=-42, y=$ 
A

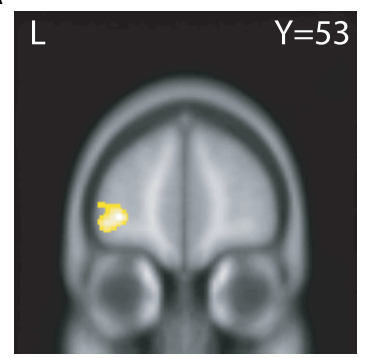

B

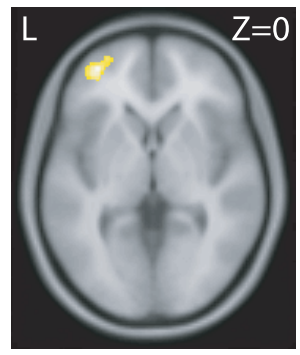

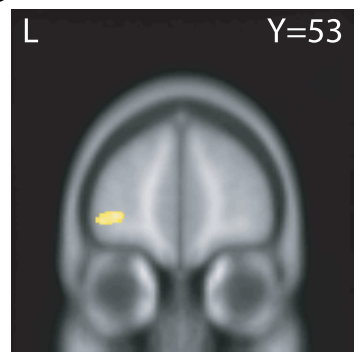

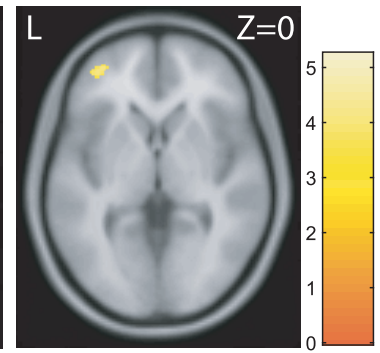

Figure 5. $A, B$, Gray matter volume of the left ventrolateral prefrontal cortex was negatively correlated with the frequency of irrationality (the number of selected bundles that violated GARP; $A$ ) and was positively correlated with the (HM index; $\boldsymbol{B})$. The frequency of irrationality and the severity of irrationality increased as gray matter volume in this region decreased. No other region was significantly correlated with economic rationality using the same analytic method. L, Left. MNI coordinates.

A

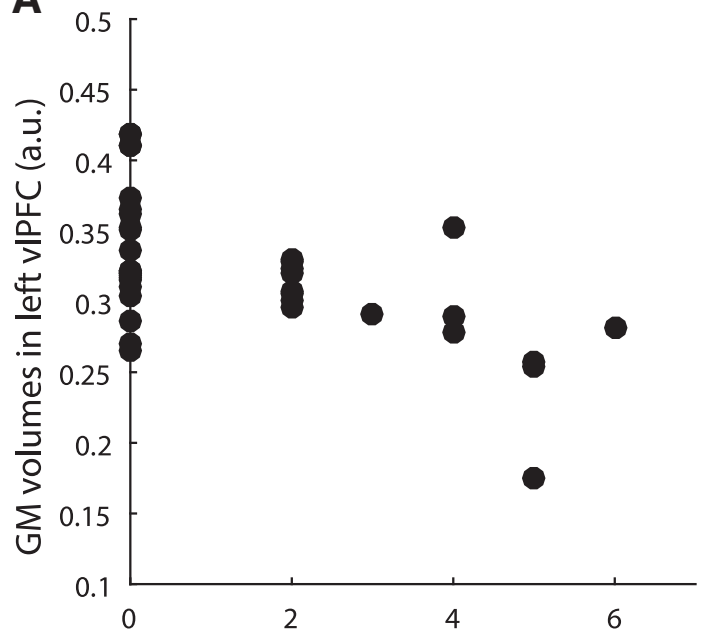

Frequency of Irrationality (GARP violations)
B

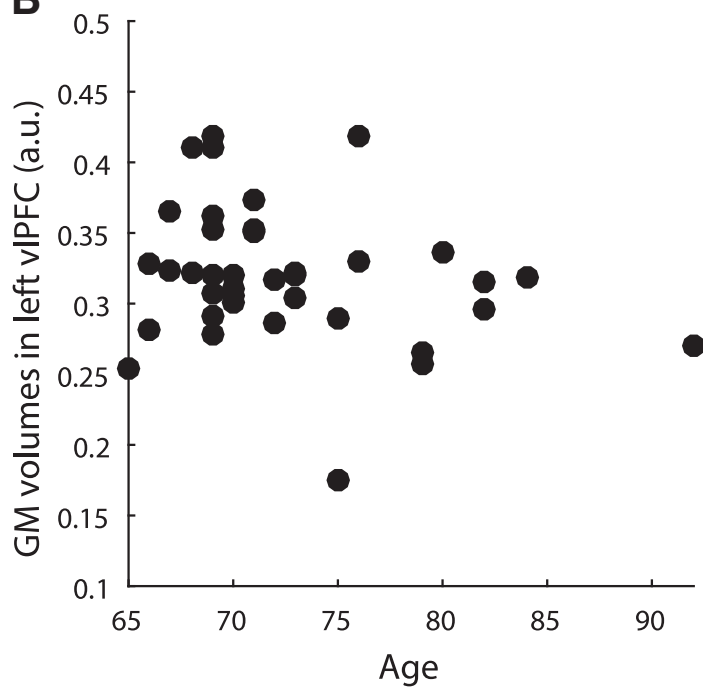

Figure 6. $\boldsymbol{A}, \boldsymbol{B}$, For each individual, gray matter volume of the left ventrolateral prefrontal cortex cluster (Fig. $5 A$ ) is plotted on the $y$-axis against the frequency of irrationality (the number of selected bundles that violated GARP on the $x$-axis $(A)$ and against age on the $x$-axis $(\boldsymbol{B})$. Note that this is presented for visualization purposes only.

$38, z=-9$; cluster number, 727; cluster level with FWE, $p=$ $0.01)$ was significantly correlated with our measure of the severity of economic irrationality (HM index; Fig. 5B) as well. Participants with lower GM volumes in this region not only made irrational decisions more frequently but also made more severe errors.

Although the whole-brain analysis showed a significant impact only on economic rationality in left vlPFC, we suspected that the right vlPFC had a less robust signal, and so it may just not be able to pass the very stringent statistical corrections. Using an ROI-based analysis, we checked whether the GM volumes of our area in the right hemisphere (right vlPFC) also showed a similar relationship with the degree of irrationality in the observed behavior. We tested it by extracting the flipped cluster from VBM analysis of frequency of irrationality in vlPFC (using MarsBar toolbox; http://marsbar.sourceforge.net/) and correlated the average GM volumes of that cluster in right vlPFC with our measure of the frequency of irrationality and the severity of irrationality separately. As we expected, we found a significant correlation with the frequency of irrationality (Pearson correlation $r_{(36)}=$ $-0.58, p<0.001$ ) and the severity of irrationality (Pearson correlation $\left.r_{(36)}=0.47, p=0.003\right)$. Participants with lower GM volumes in right vlPFC also made irrational decisions more frequently and severely. The ROI analyses of bilateral vlPFC yielded similar correlation pattern with total pairs of GARP violations and AEI. Those results are available in supplementary materials on personal website (http://decisionsrus.com/documents/ Supplementary\%20Material\%202.pdf).

Ventrolateral prefrontal connectivity: Neurosynth analysis To shed light on the possible network implications of our finding, we explored the brain circuits of economic rationality in the Neurosynth dataset. To do this, we created a reward-associated map using the Neurosynth dataset (Fig. 7). The map is strikingly similar to that in the study by Bartra et al. (2013) (meta-analytic results). In addition, we found that the left vlPFC is involved with reward-associated regions.

Next, we created a functional connectivity and meta-analytic coactivation map (Fig. 8) for our ROI, the left vlPFC, using the closest seed $(x=-36, y=52, z=-2)$ found by our VBM analysis. We found that left vlPFC coactivates with many brain areas known to play a key role in decision-making processing. The coactivation regions include ventromedial prefrontal cortex, dorsal lateral prefrontal cortex, striatum, posterior parietal cortex, and others. We used these flipped coordinates in the right hemisphere $(x=36, y=52, z=-2)$ as another seed and found that these decision-making processing areas are associated with the right vlPFC as well. 
A
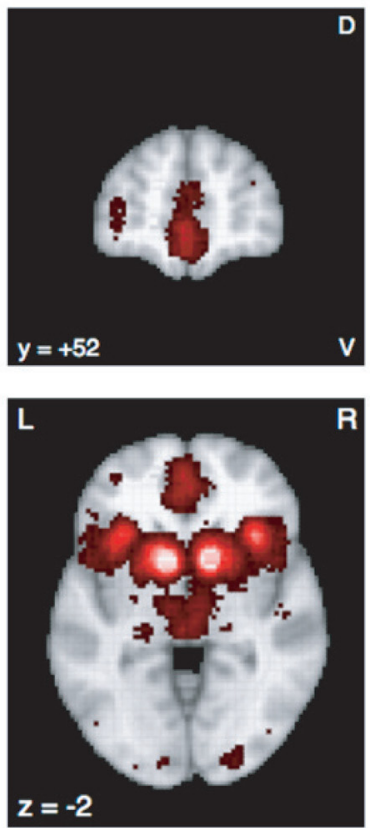

B
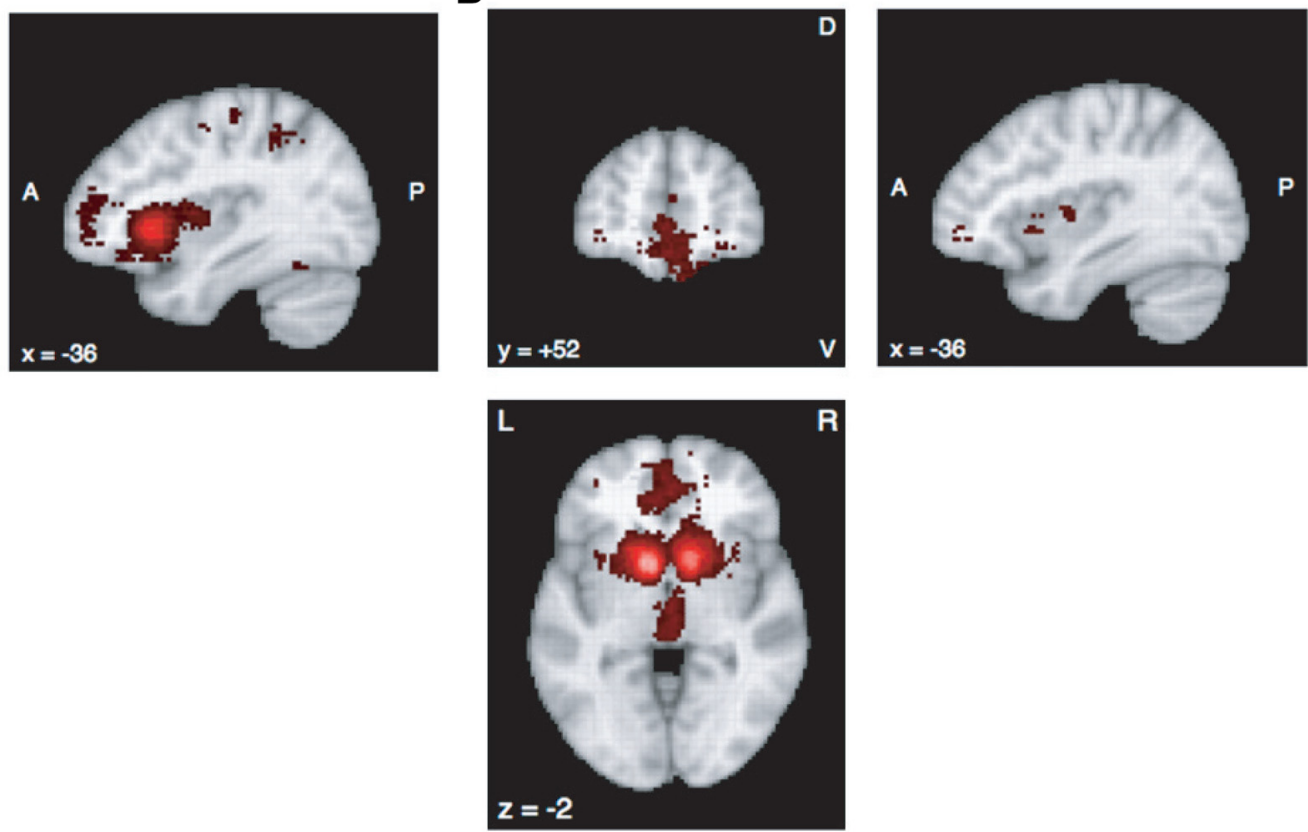

Figure 7. $\quad \boldsymbol{A}, \boldsymbol{B}$, Reward-associated regions with the key term reward using the forward inference test $(\boldsymbol{A})$ and using the reverse inference test $(\boldsymbol{B})$ from the Neurosynth by 8 June 2017 . D, Dorsal; $V$, ventral; $A$, anterior; $P$, posterior; $L$, left; $R$, right. MNI coordinates.

A
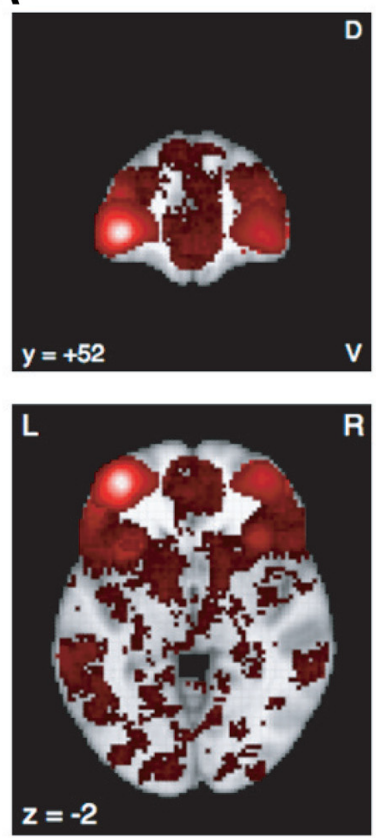

B

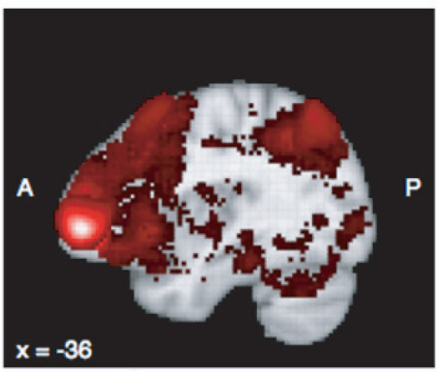

$\mathbf{R}$ $\mathbf{x}=-30$

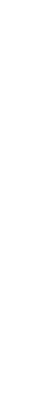

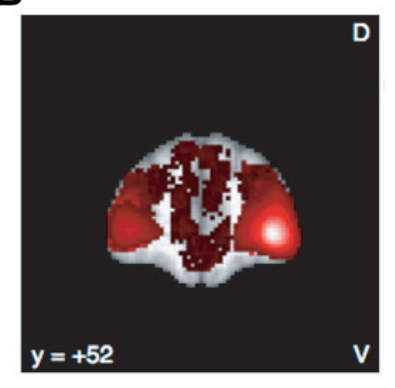
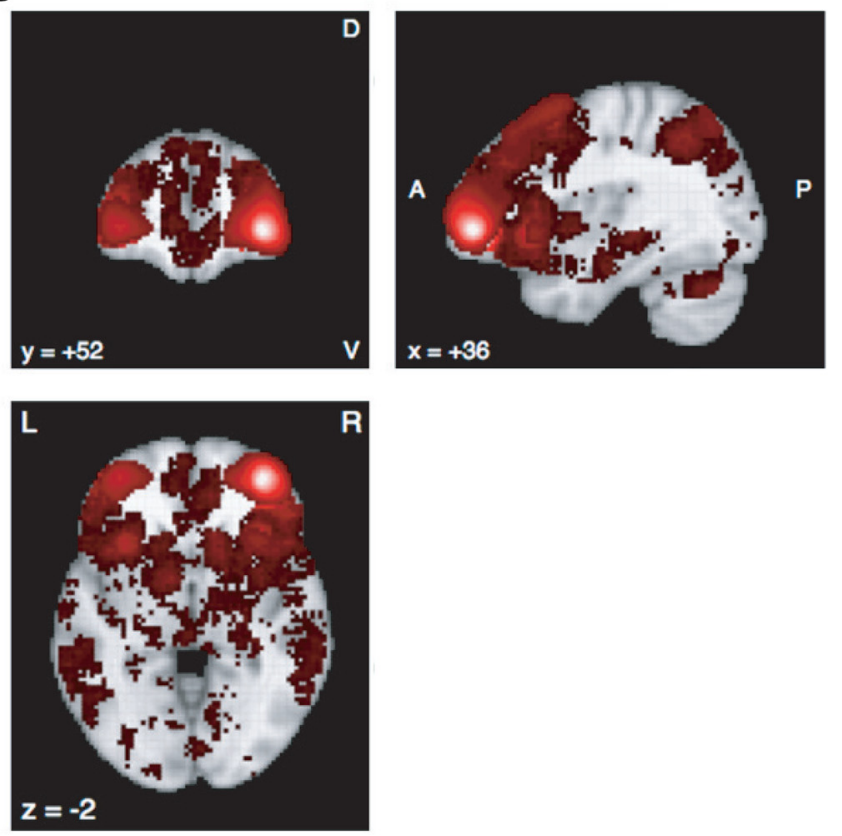

Figure 8. $\boldsymbol{A}, \boldsymbol{B}$, Functional connectivity and meta-analytic coactivation map with seed at $x=-36, y=52, z=-2(\boldsymbol{A})$ and seed at $x=36, y=52, z=-2(\boldsymbol{B})$ from the Neurosynth by 8 June 2017. D, Dorsal; $V$, ventral; $A$, anterior; $P$, posterior; $L$, left; $R$, right. MNI coordinates.

\section{Discussion}

As populations all over the world age, societies are growing concerned about the observed decline of decision-making rationality in older adulthood (Agarwal et al., 2009; Shue and Luttmer, 2009; Tymula et al., 2013). Previous articles assessing rationality in older adults used a variety of measures, as follows: propensity to choose clearly dominated options (Tymula et al., 2013); and susceptibility to choose clearly inferior financial products (Agarwal et al., 2009). From these studies, researchers concluded that on average older individuals perform worse than their younger counterparts in decision-making tasks. The reason why the quality of choice declines, even in simple tasks that do not rely on memory or complicated rules (e.g., the example in Tymula et al., 2013), is not known. Here we investigated whether older adults have a diminished ability to consistently choose their preferred alternative due to the changes in their brains. To date, there has been no test of this basic tenet of economic rationality in healthy older adults in the literature. 
In our study, we quantified the degree of rationality in 38 participants over the age of 65 years using a well known behavioral task from the economics literature. We found a higher proportion of irrational choosers in our population of older adults than among undergraduate students reported in the literature. Selecting the highest value option from a set of alternatives requires diverse capabilities, as follows: consistency in representing the utility of bundles of goods, ability to detect the highest-valued option from a set of multiple alternatives, inhibition of the irrelevant information, and ability to maintain the goal of making decisions that maximize utility (or in other words pursue a consistent goal). Although we cannot identify the mental subprocesses that cause irrationality in elders, we show here its biological roots in the structure of the vlPFC, a region that is involved in decision-making. We found that the individuals who showed a higher degree of irrationality in their behavior had less gray matter in the vlPFC. Using the Neurosynth dataset, we found that vlPFC cortex coactivates with many brain regions in the reward circuit. We therefore speculate that vlPFC is involved in some important way in the process of utility maximization.

Ventral medial prefrontal cortex (vmPFC)/medial orbital frontal cortex (not the area identified in this study) has been suggested to play a role in coding value on a common currency scale that allows individuals to compare goods that have very different units (Levy et al., 2010; Levy and Glimcher, 2011; Bartra et al., 2013). In contrast, vlPFC (the area identified in this study) has been previously shown to be selectively activated in tasks requiring decisions about higher ambiguity level lotteries (Levy et al., 2010), and it has been suggested to play a role in mechanisms for precommitment in decision-making (Crockett et al., 2013; Soutschek et al., 2017). These cognitive functions are all considered to relate to rationality, which may not only require value coding, but, depend on the ability to detect all available options, maintain stable preference, and overcome distractibility or attentional filtering. Our design was not intentionally aimed to disentangle and identify the impairments of specific cognitive functions and processes (e.g., limited attention; Masatlioglu et al., 2012) that result in rationality violations but was more limited in its scope, focusing on irrationality per se. Despite that limitation, however, we did perform a number of cognitive measures that appear to be independent of the rationality violations we observed. These included memory as measured by digit span, IQ score, and other cognitive skills. However, it remains to be investigated in future studies what specific other mental process are at the core of the rationality violations we observed to be correlated with vlPFC gray matter density.

vlPFC has also been widely studied in nonhuman primates and is often considered as a critical region in reward-learning tasks (Rich and Wallis, 2016) and object discrimination reversal tasks (Chau et al., 2015). As in our task, and more generally in the process of utility maximization that underlies all of these tasks, individuals make a decision from a menu of available actions to reach their best outcome. In a human fMRI study manipulating the value and identity of appetizing food odors in a rewardlearning task, predictive representations of identity-specific rewards were shown in the vlPFC but identity-general reward representations were shown in vmPFC (Howard et al., 2015). In line with the speculation that vlPFC is involved in choice, vlPFC showed strong positive functional coupling with vmPFC, dorsal lateral prefrontal cortex, striatum, posterior parietal cortex, and other regions in valuation systems in humans and monkeys (Neubert et al., 2015). Of course, further research will be needed to examine the causal link between vlPFC region and the decision-making process, for example by temporarily deactivating this region using noninvasive brain activation technologies.

Our finding that vlPFC plays a role in utility maximization sits well with the findings in the literature on aging. The frontal lobe, which is generally associated with higher cognitive function, is especially vulnerable to senescence. Age-related deterioration in the frontal lobe, often referred to as the "frontal aging hypothesis" (West, 1996), is believed to explain functional decline in aging. Previous studies have documented that frontal lobe is one of the brain areas showing prominent atrophy in aging (Good et al., 2001; Resnick et al., 2003), and participants whose cognitive performance declined with age have lower GM density overall in prefrontal cortex (Tisserand et al., 2004). Kievit et al. (2014) found that fluid intelligence, the ability to use different types of novel information in real time and the ability to multitask, correlate with distinct frontocortical structural properties.

Interestingly, chronological age did not correlate with the degree of irrationality in our study. This suggests that not everyone gets more irrational with age, or at least that such increases in irrationality do not happen for everybody at the same pace. This is consistent with the idea that not chronological but rather neurobiological age matters for the impairment of decision-making ability. In a similar spirit Grubb et al. (2016) have shown that gray matter volume in the right posterior parietal cortex accounts for changes in risk preferences over the lifespan better than does chronological age. Indeed, even though there is no relationship between chronological age and rationality in our sample, we found that individuals with less gray matter in the vlPFC showed more frequent and more severe economic irrationality. The relative stability of the neuroanatomy of the frontal lobe in young and midlife adults coincides with similar degrees of stability of rationality in that population (Lazzaro et al., 2016). Of course, GM reduction in healthy aging can reflect many possible changes of microstructure and cellular events, such as underlying synaptogenesis and dendritic arborization in brains of older adults (Kanai and Rees, 2011).

It is important to remember that because of the participation criteria imposed by MRI scanning and the need to come to the university to participate, we likely recruited a not truly representative sample of older adults, likely skewed toward higher functioning. This may explain the lack of the age trend in irrationality in our study. In addition, we did not attempt to measure all aspects of decision-making quality. For example, previous studies have documented that crystallized intelligence (experience and accumulated knowledge) preserves or even increases with age and may compensate for the decline of fluid intelligence (Li et al., 2013). This means that in some situations, older adults may manage as well or even better than younger adults (Bruine de Bruin et al., 2012; Li et al., 2013). However, in other situations, such as our task, that do not build upon previous experience or acquired knowledge and depend totally on subjective preference, such compensation may not occur.

Our finding that gray matter density of the vlPFC captures the limits of economic rationality advances our theoretical understanding of the decision-making process. It contributes not only to numerous studies characterizing the impact of age-related changes in brain morphometry (Good et al., 2001; Terribilli et al., 2011; Matsuda, 2013), but also more generally to the understanding of the neural mechanism of cognitive function (Denburg et al., 2005; Andrews-Hanna et al., 2007; Grady, 2012; SamanezLarkin and Knutson, 2015). The finding may contribute to our understanding of increased irrational decision-making in other conditions, for example under stress, in mental illness, and among 
addicts. For instance, severely anxious and depressed people have been shown to make more irrational choices (Weinrabe et al., unpublished results). Our results raise the possibility that measurements of gray matter density in the vlPFC may have the capacity to become a practical and useful anatomical biomarker for decision-making quality. A greater understanding of this area is needed to use this information to maintain or improve rational decision-making as we age. Moreover, it will be necessary to conduct future studies with broader populations to test whether the finding is specific to an older population only.

\section{References}

Afriat SN (1967) The construction of utility functions from expenditure data. Int Econ Rev (Philadelphia) 8:67. CrossRef

Agarwal S, Driscoll JC, Gabaix X, Laibson D (2009) The age of reason: financial decisions over the life cycle and implications for regulation. Brookings Pap Econ Act 51-117.

Afriat SN (1972) Efficiency estimation of production function. Int Econ Rev (Philadelphia) 13:568-598. CrossRef

Andreoni J, Miller J (2002) Giving according to GARP: an experimental test of the consistency of preferences for altruism. Econometrica 70:737-753. CrossRef

Andrews-Hanna JR, Snyder AZ, Vincent JL, Lustig C, Head D, Raichle ME, Buckner RL (2007) Disruption of large-scale brain systems in advanced aging. Neuron 56:924-935. CrossRef Medline

Ashburner J (2007) A fast diffeomorphic image registration algorithm. Neuroimage 38:95-113. CrossRef Medline

Ashburner J, Friston KJ (2000) Voxel-based morphometry-the methods. Neuroimage 11:805-821. CrossRef Medline

Bartra O, McGuire JT, Kable JW (2013) The valuation system: a coordinatebased meta-analysis of BOLD fMRI experiments examining neural correlates of subjective value. Neuroimage 76:412-427. CrossRef Medline

Bishop NA, Lu T, Yankner BA (2010) Neural mechanisms of ageing and cognitive decline. Nature 464:529-535. CrossRef Medline

Blair JR, Spreen O (1989) Predicting premorbid IQ: a revision of the national adult reading test. Clin Neuropsychol 3:129-136. CrossRef

Bruine de Bruin W, Parker AM, Fischhoff B (2012) Explaining adult age differences in decision-making competence. J Behav Decis Mak 25:352360. CrossRef

Burghart DR, Glimcher PW, Lazzaro SC (2013) An expected utility maximizer walks into a bar.... J Risk Uncertain 46:215-246. CrossRef Medline

Camille N, Griffiths CA, Vo K, Fellows LK, Kable JW (2011) Ventromedial frontal lobe damage disrupts value maximization in humans. J Neurosci 31:7527-7532. CrossRef Medline

Chau BK, Sallet J, Papageorgiou GK, Noonan MP, Bell AH, Walton ME, Rushworth MF (2015) Contrasting roles for orbitofrontal cortex and amygdala in credit assignment and learning in macaques. Neuron 87: 1106-1118. CrossRef Medline

Crockett MJ, Braams BR, Clark L, Tobler PN, Robbins TW, Kalenscher T, Yu C (2013) Restricting temptations: neural mechanisms of precommitment. Neuron 79:391-401. CrossRef Medline

Cuadra MB, Cammoun L, Butz T, Cuisenaire O, Thiran JP (2005) Comparison and validation of tissue modelization and statistical classification methods in T1-weighted MR brain images. IEEE Trans Med Imaging 24:1548-1565. CrossRef Medline

Denburg NL, Tranel D, Bechara A (2005) The ability to decide advantageously declines prematurely in some normal older persons. Neuropsychologia 43:1099-1106. CrossRef Medline

Eklund A, Nichols TE, Knutsson H (2016) Cluster failure: why fMRI inferences for spatial extent have inflated false-positive rates. Proc Natl Acad Sci U S A 113:7900-7905. CrossRef Medline

Folstein MF, Folstein SE, McHugh PR (1975) "Mini-mental state. A practical method for grading the cognitive state of patients for the clinician. J Psychiatr Res 12:189-198. CrossRef Medline

Giedd JN (2004) Structural magnetic resonance imaging of the adolescent brain. Ann N Y Acad Sci 1021:77-85. CrossRef Medline

Glasser MF, Coalson TS, Robinson EC, Hacker CD, Harwell J, Yacoub E, Ugurbil K, Andersson J, Beckmann CF, Jenkinson M, Smith SM, Van Essen DC (2016) A multi-modal parcellation of human cerebral cortex. Nature 536:171-178. CrossRef Medline
Glimcher PW (2010) Foundations of neuroeconomic analysis. Oxford, UK: Oxford UP.

Gogtay N, Giedd JN, Lusk L, Hayashi KM, Greenstein D, Vaituzis AC, Nugent TF 3rd, Herman DH, Clasen LS, Toga AW, Rapoport JL, Thompson PM (2004) Dynamic mapping of human cortical development during childhood through early adulthood. Proc Natl Acad Sci U S A 101:8174-8179. CrossRef Medline

Good CD, Johnsrude IS, Ashburner J, Henson RN, Friston KJ, Frackowiak RS (2001) A voxel-based morphometric study of ageing in 465 normal adult human brains. Neuroimage 14:21-36. CrossRef Medline

Grady C (2012) The cognitive neuroscience of ageing. Nat Rev Neurosci 13:491-505. CrossRef Medline

Grubb MA, Tymula A, Gilaie-Dotan S, Glimcher PW, Levy I (2016) Neuroanatomy accounts for age-related changes in risk preferences. Nat Commun 7:13822. CrossRef Medline

Gutchess A (2014) Plasticity of the aging brain: new directions in cognitive neuroscience. Science 346:579-582. CrossRef Medline

Harbaugh WT, Krause K, Berry TR (2001) GARP for kids: on the development of rational choice behavior. Am Econ Rev 91:1539-1545. CrossRef

Hayasaka S, Phan KL, Liberzon I, Worsley KJ, Nichols TE (2004) Nonstationary cluster-size inference with random field and permutation methods. Neuroimage 22:676-687. CrossRef Medline

Hedden T, Gabrieli JD (2004) Insights into the ageing mind: a view from cognitive neuroscience. Nat Rev Neurosci 5:87-96. CrossRef Medline

Houtman M, Maks J (1985) Determining all maximal data subsets consistent with revealed preference. Kwant Methoden 19:89-104.

Howard JD, Gottfried JA, Tobler PN, Kahnt T (2015) Identity-specific coding of future rewards in the human orbitofrontal cortex. Proc Natl Acad Sci U S A 112:5195-5200. CrossRef Medline

Kanai R, Rees G (2011) The structural basis of inter-individual differences in human behaviour and cognition. Nat Rev Neurosci 12:231-242. CrossRef Medline

Kievit RA, Davis SW, Mitchell DJ, Taylor JR, Duncan J, Duncan J, Henson RN, Henson RN (2014) Distinct aspects of frontal lobe structure mediate age-related differences in fluid intelligence and multitasking. Nat Commun 5:5658. CrossRef Medline

Kreps DM (1990) A course in microeconomic theory. Princeton, NJ: Princeton University Press.

Kurth F, Gaser C, Luders E (2015) A 12-step user guide for analyzing voxelwise gray matter asymmetries in statistical parametric mapping (SPM). Nat Protoc 10:293-304. CrossRef Medline

Lazzaro SC, Rutledge RB, Burghart DR, Glimcher PW (2016) The impact of menstrual cycle phase on economic choice and rationality. PLoS One 11:e0144080. CrossRef Medline

Levy DJ, Glimcher PW (2011) Comparing apples and oranges: using reward-specific and reward-general subjective value representation in the brain. J Neurosci 31:14693-14707. CrossRef Medline

Levy I, Snell J, Nelson AJ, Rustichini A, Glimcher PW (2010) Neural representation of subjective value under risk and ambiguity. J Neurophysiol 103:1036-1047. CrossRef Medline

Li Y, Baldassi M, Johnson EJ, Weber EU (2013) Complementary cognitive capabilities, economic decision making, and aging. Psychol Aging 28: 595-613. CrossRef Medline

Masatlioglu Y, Nakajima D, Ozbay EY (2012) Revealed attention. Am Econ Rev 102:2183-2205. CrossRef

Matsuda H (2013) Voxel-based morphometry of brain MRI in normal aging and Alzheimer's disease. Aging Dis 4:29-37. Medline

Neubert FX, Mars RB, Sallet J, Rushworth MF (2015) Connectivity reveals relationship of brain areas for reward-guided learning and decision making in human and monkey frontal cortex. Proc Natl Acad Sci U S A 112: E2695-E2704. CrossRef Medline

Ofstedal MB, Fisher GG, Herzog AR (2005) HRS/AHEAD documentation report. Documentation of cognitive functioning measures in the health and retirement study. HRS Documentation Report DR-006. Ann Arbor, MI: Survey Research Center, University of Michigan.

Resnick SM, Pham DL, Kraut MA, Zonderman AB, Davatzikos C (2003) Longitudinal magnetic resonance imaging studies of older adults: a shrinking brain. J Neurosci 23:3295-3301. Medline

Rich EL, Wallis JD (2016) Decoding subjective decisions from orbitofrontal cortex. Nat Neurosci 19:973-980. CrossRef Medline

Richter MK (1966) Revealed preference theory. Econometrica 34:635. CrossRef Samanez-Larkin GR, Knutson B (2015) Decision making in the ageing 
brain: changes in affective and motivational circuits. Nat Rev Neurosci 16:278-289. CrossRef Medline

Samuelson PA (1938) A note on the pure theory of consumer's behaviour. Economica 5:61. CrossRef

Shipley WC (1940) A self-administering scale for measuring intellectual impairment and deterioration. J Psychol 9:371-377. CrossRef

Shue K, Luttmer EFP (2009) Who misvotes? The effect of differential cognition costs on election outcomes. Am Econ J Econ Policy 1:229-257. CrossRef

Soutschek A, Ugazio G, Crockett MJ, Ruff CC, Kalenscher T, Tobler PN (2017) Binding oneself to the mast: stimulating frontopolar cortex enhances precommitment. Soc Cogn Affect Neurosci 12:635-642. CrossRef Medline

Terribilli D, Schaufelberger MS, Duran FL, Zanetti MV, Curiati PK, Menezes PR, Scazufca M, Amaro E Jr, Leite CC, Busatto GF (2011) Age-related gray matter volume changes in the brain during non-elderly adulthood. Neurobiol Aging 32:354-368. CrossRef Medline

Tisserand DJ, van Boxtel MP, Pruessner JC, Hofman P, Evans AC, Jolles J (2004) A voxel-based morphometric study to determine individual differences in gray matter density associated with age and cognitive change over time. Cereb Cortex 14:966-973. CrossRef Medline

Tymula A, Rosenberg Belmaker LA, Ruderman L, Glimcher PW, Levy I (2013) Like cognitive function, decision making across the life span shows profound age-related changes. Proc Natl Acad Sci U S A 110: 17143-17148. CrossRef Medline
Uttl B (2002) North American adult reading test: age norms, reliability, and validity. J Clin Exp Neuropsychol 24:1123-1137. CrossRef Medline

Varian HR (1982) The nonparametric approach to demand analysis. Econometrica 50:945-973. CrossRef

Varian HR (1996) Computational economics and finance: modeling and analysis with Mathematica. New York: Springer.

Warshall S, Stephen (1962) A theorem on Boolean matrices. J ACM 9:11-12. CrossRef

Wechsler D (1997) WAIS-III: Wechsler adult intelligence scale. San Antonio, TX: Psychological Corporation.

Weinrabe A, Chung H-K, Tymula A, Hickiea I (2017) Economic rationality in youth with emerging mood disorders; evaluating the impact of disregulated affect and emotion in adolescents using economic decisionmaking tools, in press.

West RL (1996) An application of prefrontal cortex function theory to cognitive aging. Psychol Bull 120:272-292. CrossRef Medline

Yarkoni T, Poldrack RA, Nichols TE, Van Essen DC, Wager TD (2011) Large-scale automated synthesis of human functional neuroimaging data. Nat Methods 8:665-670. CrossRef Medline

Zachary RA, Shipley WC (1986) Shipley institute of living scale: revised manual. Torrance, CA: Western Psychological Services.

Zhang Y, Brady M, Smith S (2001) Segmentation of brain MR images through a hidden Markov random field model and the expectationmaximization algorithm. IEEE Trans Med Imaging 20:45-57. CrossRef Medline 